University of Nebraska - Lincoln

DigitalCommons@University of Nebraska - Lincoln

Papers in Materials Science

Chemical and Biomolecular Engineering

Research and Publications

5-1-1995

\title{
Simulation of the growth of CVD films
}

Jacob J. Thiart

Department of Chemical Engineering, Suny at Buffalo, Amhearst , 14260

Vladimir Hlavacek

Department of Chemical Engineering, State university of New York, Buffalo. NY

Hendrik J. Viljoen

University of Nebraska-Lincoln, hviljoen1@unl.edu

Follow this and additional works at: https://digitalcommons.unl.edu/chemengmatsci

Part of the Materials Science and Engineering Commons

Thiart, Jacob J.; Hlavacek, Vladimir; and Viljoen, Hendrik J., "Simulation of the growth of CVD films" (1995). Papers in Materials Science. 8.

https://digitalcommons.unl.edu/chemengmatsci/8

This Article is brought to you for free and open access by the Chemical and Biomolecular Engineering Research and Publications at DigitalCommons@University of Nebraska - Lincoln. It has been accepted for inclusion in Papers in Materials Science by an authorized administrator of DigitalCommons@University of Nebraska - Lincoln. 


\section{Simulation of the growth of CVD films}

\section{INTRODUCTION}

Chemical vapor deposition (CVD) is the preferred method of manufacture for solid films used in many industrially important thin and thick film applications. Requirements for the physical, mechanical and electrical properties of these films are becoming increasingly difficult to achieve, and deposition morphology plays an important role in this regard. Recently, we proposed a continuum model describing the evolution of a gas-solid interface during atmospheric pressure CVD (Viljoen er al.. 1994). A linear stability analysis (LSA) was used to determine the effect of reactor conditions on planar growth stability. The present paper discusses numerical solution of this model, and uses simulation examples to illustrate interface evolution under typical deposition conditions and from arbitrary initial interface shapes.

\section{MODEL FORMULATION}

We consider the growth of an amorphous solid film during a typical high pressure (0.1-1 atm), low Knudsen number $(K n \ll 1)$ CVD process. Transport of gaseous species to the interface is assumed to be diffusive. Details of the model derivation are given in Viljoen $\boldsymbol{e t}$ al. (1994) and only the final dimensionless governing equations are presented here. The gas phase concentration balance becomes:

$$
\frac{\partial \mathscr{C}}{\partial \tau}=\frac{1}{P e}\left(\frac{\partial^{2} \mathscr{C}}{\partial \xi^{2}}+\frac{\partial^{2} \mathscr{C}}{\partial \zeta^{2}}\right)+\frac{\partial \mathscr{C}}{\partial \zeta}
$$

and the interface evolution equation (solid phase):

$$
\frac{\partial H}{\partial \tau}=\frac{\rho}{P e}(\nabla \mathscr{C} \cdot \mathrm{n}) \sqrt{1+H_{\xi}^{2}}-1+\chi\left[\frac{\kappa_{\xi}}{\sqrt{1+H_{\xi}^{2}}}\right]_{\xi}
$$

with boundary conditions

$$
\begin{gathered}
\mathscr{C}=1 \quad \text { at } \zeta=\frac{9}{L^{\prime}} \\
\left.\frac{\partial \mathscr{C}}{\partial \xi}\right|_{\xi=0}=\left.\frac{\partial \mathscr{C}}{\partial \xi}\right|_{\xi=n},
\end{gathered}
$$

$$
\mathscr{Q}(0, \zeta)=\mathscr{C}(n \ell, \zeta) \quad \text { where } \mathrm{n}=0, \pm 1, \pm 2, \ldots
$$

$$
\frac{1}{P e}(\nabla \mathscr{C} \cdot \mathbf{n})=\vartheta\left(1-\frac{\Gamma}{L} \kappa\right) \mathscr{C} \text { at } \zeta=H
$$

and

$$
\begin{aligned}
H(0) & =H(n \ell) \\
H_{\xi}(0) & =H_{\xi}(n \ell) \\
H_{\xi \xi}(0) & =H_{\xi \xi}(n \ell), \\
H_{\xi \xi \xi}(0) & =H_{\xi \xi \xi}(n \ell), \quad \text { where } \mathrm{n}=0, \pm 1, \pm 2, \ldots
\end{aligned}
$$

and where

$$
\kappa=-\frac{H_{\xi \xi}}{\left(1+H_{\xi}^{2}\right)^{3 / 2}}, P e=\frac{V_{0} L}{D_{f}}, \vartheta=\frac{k K_{A D}}{V_{0}} .
$$

Note that the period over which changes are considered is d. The value of Pe is extremely small for most cases since film growth occurs at such a low rate. Another important parameter is the Damkohler number, $D a$, which we can define as $k K_{A D} S / D_{f}$. It gives an indication of the relative magnitude of gas diffusional and surface kinetic resistance. In our system a Damkohler number $D a \ll$ I represents kinetic control and practically no gas diffusional resistance, while $D a \gg 1$ corresponds to deposition in the severely diffusion-limited regime. Note that the Damkohler number we define here is equivalent to the so-called CVD number defined by Van den Brekel and Jansen (1977). They used it to characterize the state of the deposition process, after their experiments of Si deposition showed that deposition uniformity decreased with increasing values of the CVD number. Our simulation examples will show similar phenomena.

\section{SOLUTION OF GOVERNING EQUATIONS}

A previous study (Viljoen et al., 1994) showed that valuable information about a deposition process can be obtained from a linear stability analysis (LSA), without solving the full system of equations. The LSA is used to predict system conditions where planar film growth and thus film uniformity can be ensured. However, it is also important to be able to predict film morphology by solving the governing equations under different sets of operating conditions and from arbitrary initial conditions.

The governing equations represent a set of coupled, nonlinear partial differential equation ( $P D E \mathrm{~s}$ ), which need to be solved numerically. A time scale of change can be identified for each phase: $\tau_{g}=L^{2} / D_{f}$ is the characteristic time for gas diffusion and $7,=L / V_{0}$ the characteristic time for interface evolution. The ratio of these characteristic times gives an indication of the time scale on which to solve the problem. This ratio is given by $\tau_{g} / \tau_{s} \equiv \boldsymbol{P}$. Since $P e \ll 1$ for all cases we will consider, it means that the gas phase is in pseudo steady state with respect to growth in the solid phase. This greatly simplifies the solution procedure. Instead of solving the governing equations simultaneously, evolution of the interface can be followed on the time scale of solid growth and at each instant in time the steady state gas phase problem can be solved independently.

The gas phase problem was solved with e.g. the Galerkin Finite Element Method, using quadrilateral elements and bilinear basis functions. For the following interface evolution, we discretized the horizontal coordinate into an equidistant grid and approximated spatial derivatives with finite difference formuli. This transforms the problem into a set of ordinary differential equations (ODEs), for which we used an 
explicit solution method. Stability requirements dictate a small time step, and the maximum time step can be determined'with a formula similar to that defined for parabolic equations [see e.g. Finlayson (1980)]. In our case, however, spatial derivatives up to fourth order are present in the surface diffusion term. This is not common in chemical engineering applications, where typically reaction-diffusion or convection-diffusion problems are encountered. Higher derivatives tend to amplify the effect of kinks in the interface. Computational problems can usually be avoided by flooding problem areas with many points. Unfortunately, this decreases the maximum allowable time step. For example, if the number of points are doubled, the time step has to be reduced about sixteen times. For time integration a fourth order Runge-Kutta method with automatic time step adjustment was used.

\section{NUMERICAL EXAMPLES}

With periodic boundary conditions, one can in principle choose any length of substrate on which to study deposition. However, if this length is too small, the results may not be representative of a real case. The horizontal length is therefore chosen 5 to 20 times larger than the wavelength associated with the "most" unstable perturbation. The latter is the perturbation associated with the largest eigenvalue in the LSA under the specific conditions [see Viljoen et al. (1994)] Consider a CVD process with parameters in the range typical for the CVD growth of e.g. $\mathrm{SiC}$ and $\mathrm{Cr}_{2}$, thus atmospheric pressure and temperatures higher than $900 \mathrm{~K}$ Typical parameter values are given in Table 1. For these parameters and at a temperature of $1100 \mathrm{~K}$ the wavelength of most unstable perturbation (as determined from LSA) is $85.2 \mathrm{pm}$. Therefore, an acceptable horizontal length to choose as solution domain is $500 \mu \mathrm{m}$.

Another important characteristic is the following: We can use the LSA to determine the time needed to double the amplitude of a protrusion with most unstable wavelength, which is the dominant one at short deposition times. At $1100 \mathrm{~K}$ this time is $142 \mathrm{~h}$, which means that in the typical duration of deposition we will not observe a significant change in planar morphology even though planar deposition is unstable under those conditions. This situation changes drastically for deposition at $1600 \mathrm{~K}$, where the wavelength of most unstable perturbation is $7.6 \mathrm{pm}$. The time to double the amplitude is now only $140 \mathrm{~s}$, which means very significant changes in the morphology will take place in normal operating times.

Now consider a case where the substrate has a random initial roughness onto which a film is deposited. The effect of temperature and Damkohler number on the morphology of film growth is shown in Fig. 1. Note that the duration of each run is different, since the deposition rate increases with increasing Damköhler number. The morphology of the films grown at $D a=1 / 20$ and $D a=1$ is very similar, but both cases are quite different than the run for $D a=20$. At low to moderate Damkohler numbers the initial randomly rough surface develops into a characteristic nodular structure,

Table 1. System parameters for typical CVD process

\begin{tabular}{cc}
\hline Parameter & Value \\
\hline$s(\mathrm{~m})$ & $10^{-3}$ \\
$\delta(\mathrm{m})$ & $10^{-10}$ \\
$k K_{A D}(\mathrm{~m} / \mathrm{s})$ & $2 \times 10^{7} \exp [-24056 / T]$ \\
$D_{f}\left(\mathrm{~m}^{2} / \mathrm{s}\right)$ & $10^{-10} T^{1.823}$ \\
$D_{e}\left(\mathrm{~m}^{4} / \mathrm{s}\right)$ & $\exp [-2000 / T(5.0+0.0033 T)]$ \\
& \\
$\Gamma(\mathrm{m})$ & $8 \times 10^{-9} / T$ \\
\hline
\end{tabular}
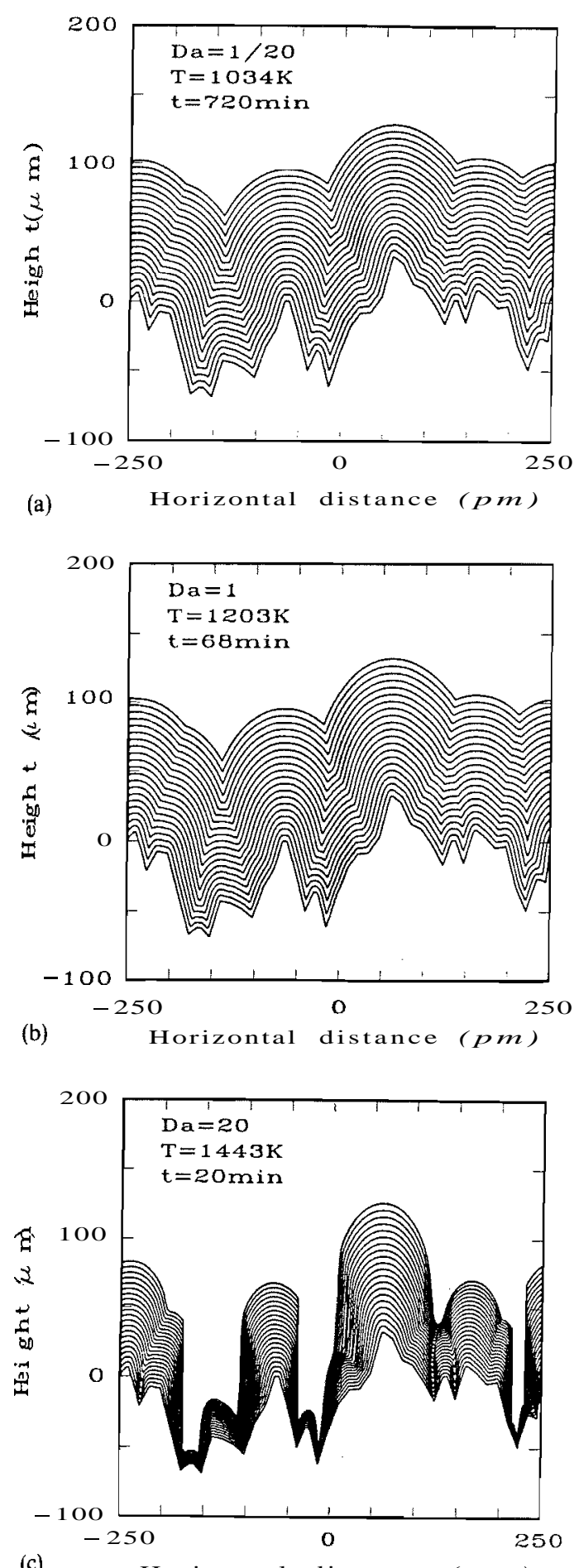

Horizontal distance $(p m)$

Fig. 1. Effect of temperature and thus Damkohler number on uniformity of film growth at a constant pressure of $1 \mathrm{~atm}$ : (a) $D a=1 / 20$, (b) $D a=1$, (c) $D a=20$.

which is commonly observed in CVD experiments (Vandenbulcke and Vuillard, 1976; Chin et al., 1977; Schmidt, 1991). The results indicate that larger nodules grow at the expense of smaller ones. This growth-death phenomenon is commonly observed in deposition processes (Messier, 1986). It 


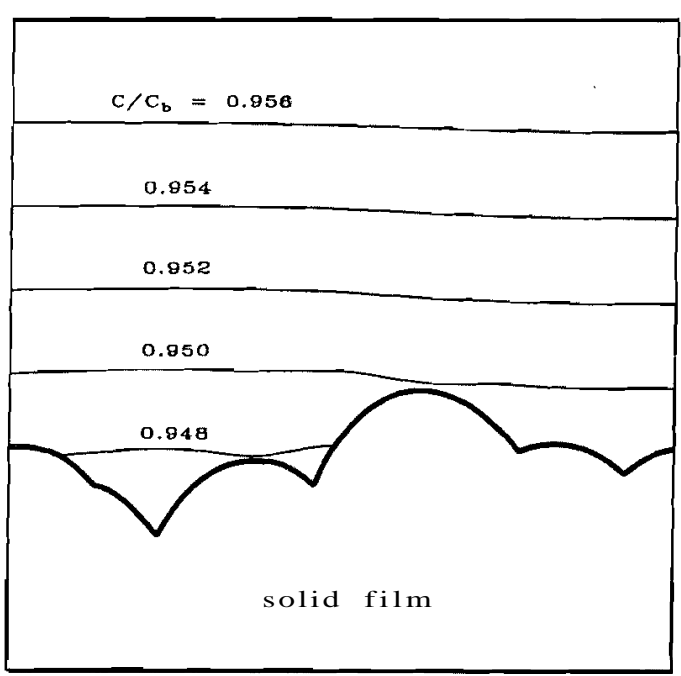

(a)

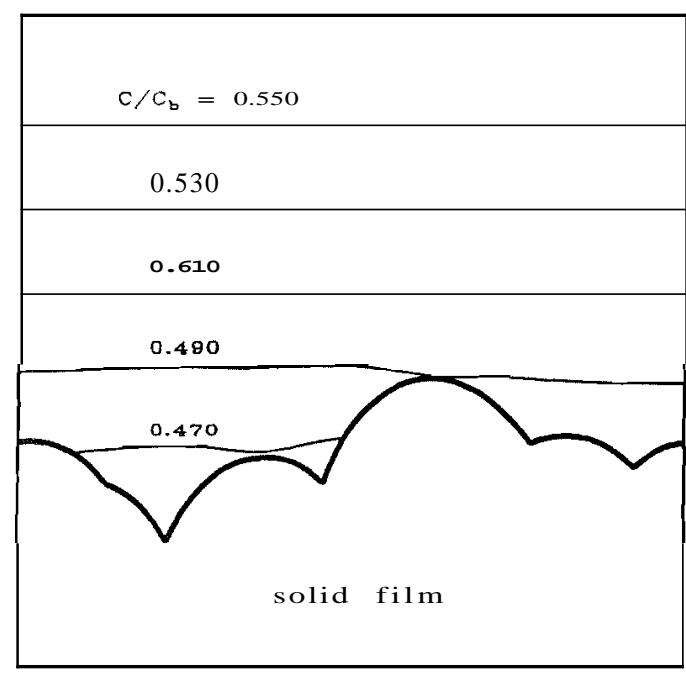

(b)

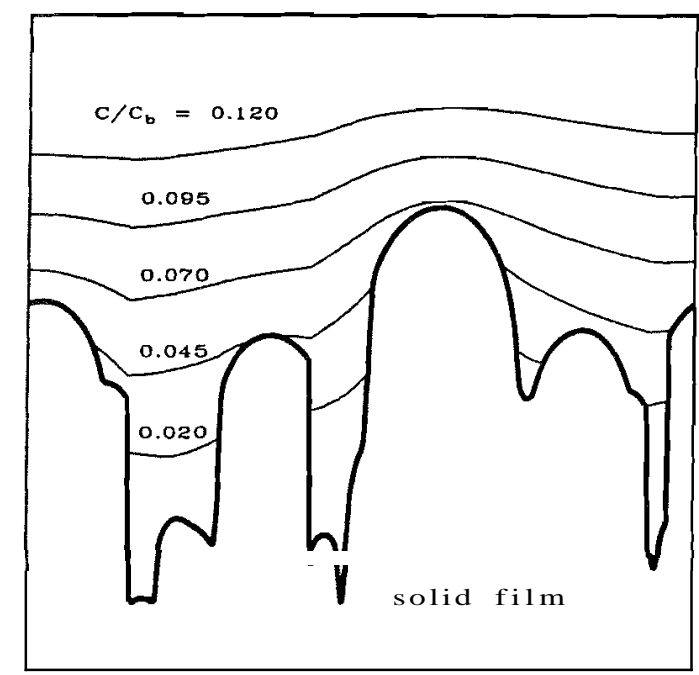

(c)

Fig. 2. Iso-concentration lines for deposition conditions in Fig. 1. has been shown that the dominant nodule size follows a power law relation with respect to the film thickness, thus $\mathrm{D},=t^{e}$, where $\mathrm{D}$, is the dominant nodule size, $t$ the time of deposition or film thickness, and e an exponent which depends on deposition conditions. For the run at $\mathrm{Da}=1$ the exponent is found to be $\mathrm{e}=0.64$, which compares well with

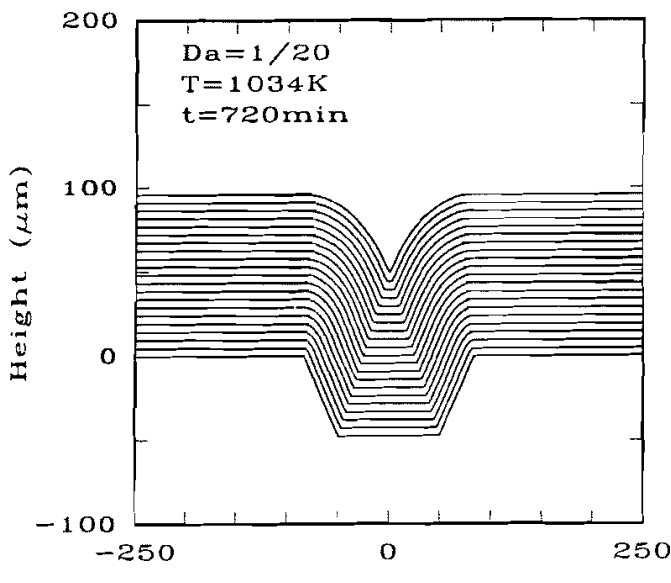

(a) Horizontal distance $(\mu \mathrm{m})$

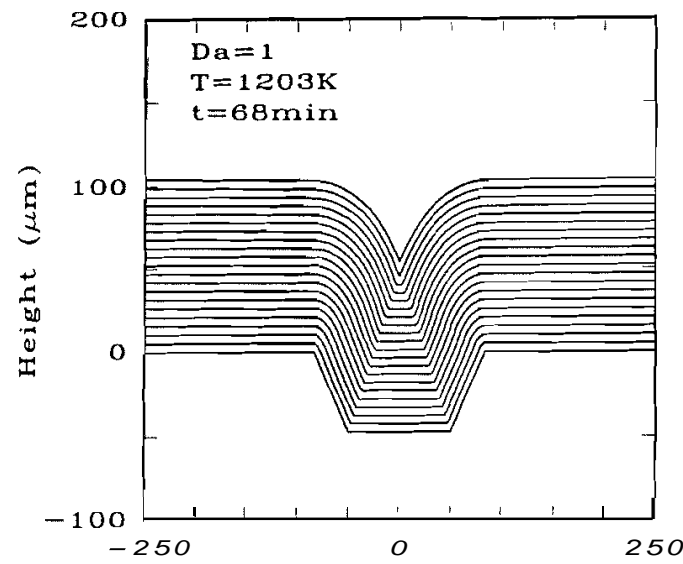

(b) Horizontal distance $(p m)$

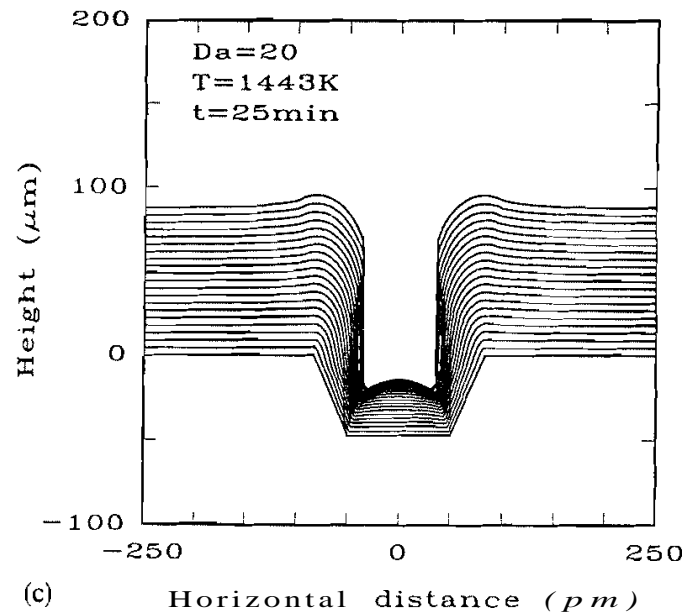

Fig. 3. Effect of temperature and thus Damkohler number on step coverage in a trench at a constant pressure of $1 \mathrm{~atm}$ : (a) $D a=1 / 20$, (b) $D a=1$, (c) $D a=20$. 
the predicted value of Kardar $t$ t al. (1986). They solved a simplified growth model analytically and found the exponent to be $e=2 / 3$.

At $\mathrm{Da}=20$ interface growth does not seem to follow the typical growth-death phenomenon. Nodules tend to develop

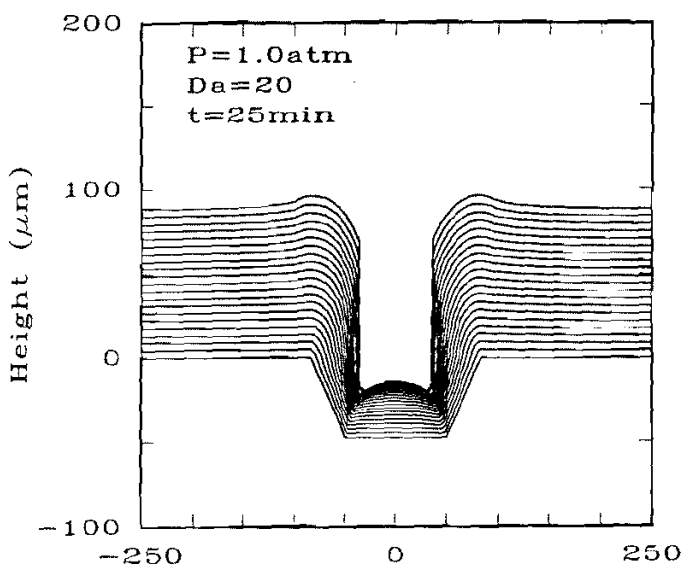

(a) Horizontal distance ( $\mu \mathrm{m})$

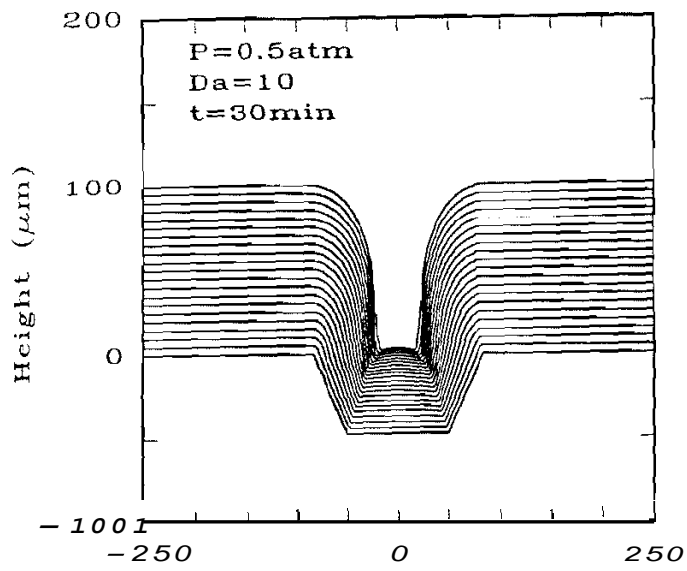

(b)

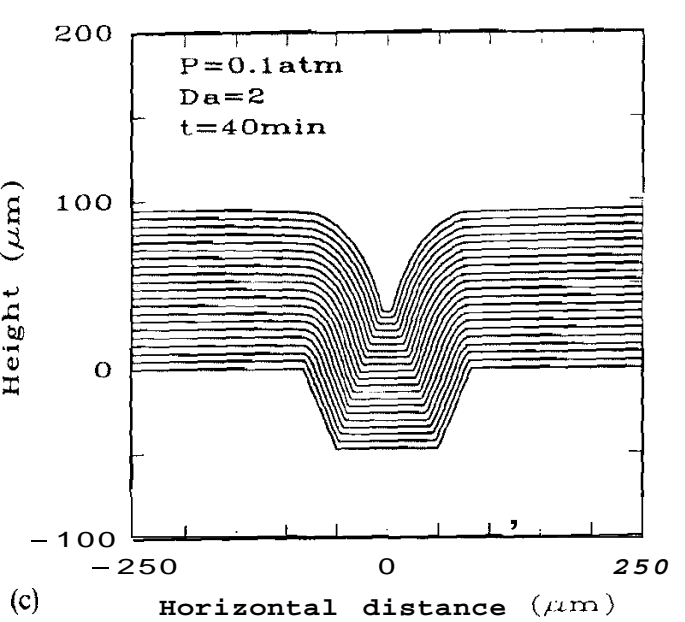

Fig. 4. Effect of reactor pressure on step coverage of a trench under diffusion-limited conditions: (a) $\mathrm{P}=1.0 \mathrm{~atm}$, (b) $\mathrm{P}=$ $0.5 \mathrm{~atm}$, (c) $\mathrm{P}=0.1 \mathrm{~atm}$. into long fingurs with deep groves between them. This has been observedexperimentally in the CVD growth of Si from $\mathrm{SiHCl}_{3}$ in the sas phase (van den Brekel, 1977) under diffusion-limited conditions. It is also similar to dendritic growth observed in solldification experiments where evolution of the liquid-solid iruerface is driven by diffusion of heat (Huang and Glicksman, 1981a, b). The formation of grooves is caused by deplation of reactant under diffusion-limited conditions. Note te tendency of some fingers to nose ahead of their neighbors. This is clear from Fig. 1(c), where reactant at the tip of the second nodule (at about the $-140 \mu \mathrm{m}$ position) is depleted and growth therefore inhibited. Iso-concentration lines the runs mentioned above are shown in Fig. 2 . Note how tie reactant concentration close to the interface changes with is close to the walue in the hulk and at $\mathrm{Da}=20$ it is close to zero.

When fings: formation takes place, gradients of the interface $(\partial H / \partial \xi)$ a diong the sides of fingers become very large and can in fact aporoach infinity. The growth model represents height of the interface $\mathrm{H}$ as a single-valued function of horizontal comrdinate $\zeta$, and it is therefore assumed $\hat{\partial} H / \hat{c} \xi \xi$ is finite. As a result, computational problems are encountered when gradien become very large. To solve this problem. one can reforaulate the model in a parametric form, where a different parameter than the horizontal coordinate is used. This will be addressed elsewhere.

Now consider a case where the initial interface is in the form of a duc or trench. This shape is typically found in the electronics industry, but the length scale may be quite different. It is often required that the thickness on all sides of the trench be the same. This requirement can be expressed in terms of the sen coverage (Oh et al. 1992). which represents the ratio of fim thickness in the bottom of the trench to that at the top of the trench. The effect of temperature and thus Damköhler namber on the film growth in a trench is shown in Fig. 3. Doposition parameters and physical properties were the same as in Table 1 and $500 \mathrm{pm}$ of the substrate was considered. The results clearly illustrate that step coverage decreases wits increasing Damkohler number. which is similar to decreare in film uniformity observed in the previous example.

The runs in Fig. 3 were conducted at a constant pressure of $1 \mathrm{~atm}$. Suprose one wants to maintain the high deposition temperature fut improve step coverage at that temperature by adjusting other parameters, such as reactant dilution ratio or pressare. At such a high temperature the deposition is diffusion-limited, so step coverage should improve if the diffusional limitation can be decreased. Gas diffusion is inversely relatad to reactor pressure, which means step coverage should improve if the reactor pressure is decreased at constant dilusion ratio. Three runs at different pressure and constant temperature of $1443 \mathrm{~K}$ were performed and the results are shown in Fig. 4. The graphs indicate that the step coverage indsed improves. Note that the Damkohler number decreases linearly with decreasing pressure.

\section{CONCLUSIONS}

Numericalsalution of a continuum model derived for the evolution of $\approx$ gas-solid interface during atmospheric pressure CVD, is described. Simulation results showed that deposition monghology is strongly dependent on the Damköhler number, A significant decrease in film uniformity was observed forincreasing values of the $D a$. This trend was also observed for growth in a trench, where step coverage was found to degease with increasing values of Da. Under diffusion-limited conditions, step coverage was found to improve if the diffusieral limitations could be decreased by decreasing system pressure at constant substrate temperature. Thr numerical results suggest that film uniformity and optimal step coverage can be achieved by changing operating condi- 
tions in such a way that the lowest value of a Damköhler number of deposition is achieved.

JACOB J. THIART, VLADIMIR. HLAVACEK ${ }^{\dagger}$

Laboratory of Ceramic and Reaction Engineering

Department of Chemical Engineering

C.C. Furnas Hall

State University of New York at Buffalo

Amherst, NY 14260, U.S.A.

HENDRIK J. VILJOEN

Department of Chemical Engineering

University of Nebraska at Lincoln

Lincoin, NE 68588-0126, U.S.A.

\section{NOTATION}

L dimensionless concentration, subscript: b-bulk

$D$ diffusion coefficient, subscripts: $f$-gas, $\mathrm{m}^{2} / \mathrm{s}$; $e$-surface, $\mathrm{m}^{4} / \mathrm{s}$

Da Damköhler number, defined as $k K_{A D^{s}} / D_{f}$ or $P e \vartheta S / L$

$H$ dimensionless height of film

$k K_{A D} \quad$ rate constant, $\mathrm{m} / \mathrm{s}$

$\ell$ dimensionless length of film considered, period

$L \quad$ characteristic length scale, $\sqrt{D_{e} / \delta V_{0}}, \mathrm{~m}$

$P_{e} \quad$ Peclet number, $V_{0} L / D_{f}$

$s \quad$ boundary layer thickness, $m$

$V_{0} \quad$ reference deposition rate, $\mathrm{m} / \mathrm{s}$

Greek letters

$\gamma \quad$ molar volume, $\mathrm{m}^{3} /$ moje

$\stackrel{\gamma}{\Gamma} \quad$ capillary length, $m$

$\delta$ radius of molecule, $m$

$\zeta$ vertical coordinate, dimensionless

$\vartheta$ dimensionless parameter, defined as $k K_{A D} / V_{0}$

$\kappa \quad$ dimensionless surface curvature,

$$
-\frac{H_{\xi \xi}}{\left(1+H_{\xi}^{2}+H_{n}^{2}\right)^{3 / 2}}
$$

horizontal coordinate, dimensionless

dimensionless parameter, equivalent to $\gamma C_{b}$

dimensionless time, $t V_{0} / L$

$\chi \quad$ dimensionless parameter, equivalent to $D_{e} / V_{0} L^{3}$

\section{- REFERENCES}

Chin, J., Gantzel, P. K. and Hudson, R. G., 1977, The structure of chemical vapor deposited silicon carbide. Thin Solid Films 40, 57-72.

Finlayson, B. A., 1980, Nonlinear Analysis in Chemical Engineering. McGraw-Hill, New York.

Huang, S.-C. and Glicksman, M. E., 1981a, Fundamentals of dendritic solidification I: Steady state tip growth. Acta Metal. 29, 701-715.

Huang, S.C. and Glicksman, M. E., 1981b, Fundamentals of dendritic solidification II: Development of sidebranch structure. Acta Metal. 29, 717-734.

Kardar, M., Parisi, G. and Zhang, Y.-C., 1986, Dynamic scaling of growing interfaces. Phys. Rev. Lett. 56 (9), 889-892.

Messier, R., 1986, Toward quantification of thin film morphology. J. Vac. Sci. Technol. A, 4 (3), 490-495.

Oh, H. J., Rhee, S. W. and Kang, I. S., 1992 Simulation of CVD process by boundary integral technique. J. electrochem. Soc. 139 (6), 1714-1720.

Schmidt, W. U., 1991, The development of chromium diboride fibers by CVD for use as reinforcement material in

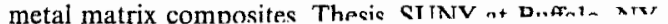

